\title{
Oral carcinoma cuniculatum: an unacquainted entity with diagnostic challenges - a case report
}

\author{
Safaa Baz ${ }^{1 *}$ (D) Hatem Wael Amer² and Ali A. Wahed ${ }^{2}$
}

\begin{abstract}
Background: Oral carcinoma cuniculatum (OCC) is an unacquainted well-differentiated subtype of oral squamous cell carcinoma, which displays unique clinic-pathological features. Up to date, OCC remains rare with about 75 reported cases, and is frequently missed or even misdiagnosed.

Case presentation: The aim of the present work was: to report a case of OCC in mandibular gingiva and to highlight its main clinic-pathological diagnostic features: with an exophytic cobble-stone surface and a characteristic endophytic burrowing architecture, as well as to differentiate between it and other closely similar lesions including verrucous carcinoma, papillary squamous cell carcinoma, and well-differentiated conventional oral squamous cell carcinoma.

Conclusions: An accurate diagnosis of OCC entails awareness of the clinicians and pathologists about its entity, proper knowledge of the diagnostic clinical and histopathological evidence, and the ability to differentiate it from closely similar lesions.
\end{abstract}

Keywords: Oral carcinoma cuniculatum, Cunniculate carcinoma, Rabbit burrows, Verrucous carcinoma, Oral squamous cell carcinoma, Differential diagnosis, Case report

\section{Background}

Carcinoma cuniculatum (CC), called cuniculate carcinoma, is a rare low-grade carcinoma [1]. Arid et al. reported a case series of $\mathrm{CC}$ on the plantar surface of the foot for the first time [2]. CC has been recorded in other sites including the abdominal wall, skin and, genital region [3-6]. However, in the oral cavity CC was first identified by Flieger and Owinski [7]. A plethora of synonyms were used in the past denoting this entity such as epithelioma cuniculatum, Buschke-Lowenstein tumor, and inverted verrucous carcinoma $(\mathrm{VC})[1,2,5]$.

*Correspondence: safaa.baz@bue.edu.eg

${ }^{1}$ Oral Pathology Department, Faculty of Dentistry, British University in Egypt (BUE), El Sherouk City, Suez Desert Road, P.O. Box 43, Cairo 11837,

Egypt

Full list of author information is available at the end of the article
In terms of its unique clinical and pathological aspects, the World Health Organization (WHO) declared CC of the oral cavity, called oral carcinoma cuniculatum (OCC), as a separate well-differentiated subtype of oral squamous cell carcinoma (OSCC) in 2005 and 2017. Also, OCC and VC were considered as different subtypes, although of being confused in the past $[1,8]$. OCC remains a rare entity that has diagnostic challenges, with a total of about 75 cases between 1977 and May 2021 were described in the literature [9]. Herein, we present a new case of OCC in the gingiva of an elderly female.

\section{Case presentation}

An 86-year-old female patient presented with a 6 months history of non-painful lesion involved her left lower gingiva. A thorough medical history and clinical data were obtained. On intra-oral examination, a firm mandibular gingival swelling with blunt, pebbly 


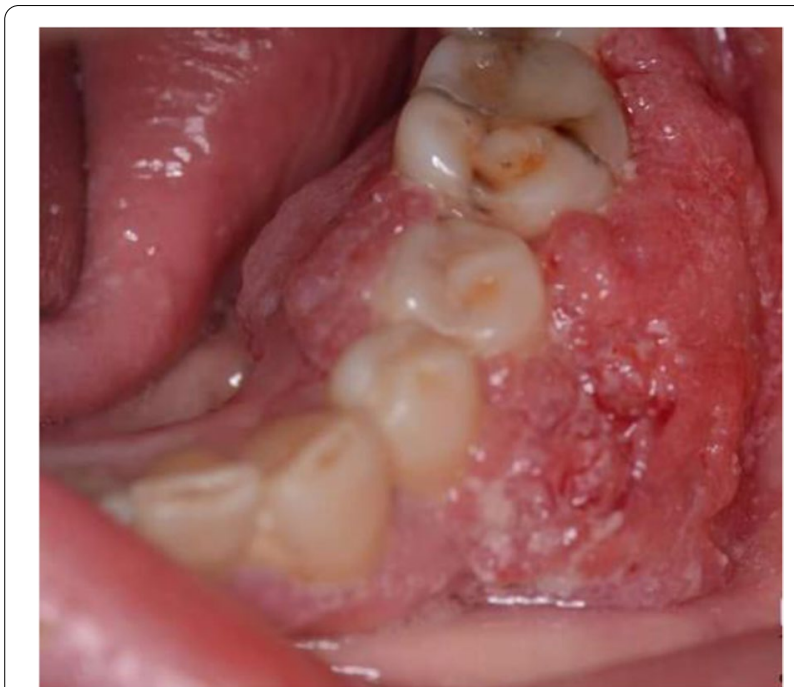

Fig. 1 Clinical presentation of a pinkish and red-white colored lesion with an exophytic"cobble-stone" surface "cobble-stone" surface was developed on both the buccal and lingual aspects of the premolar-molar region. The color of the lesion was similar to the surrounding normal mucosa intermixed with red-white areas (Fig. 1). No palpable draining lymph nodes were noted. Upon preoperative radiographic evaluation, no evidence of bone involvement was noted.

An incisional biopsy specimen (measuring $1.5 \times 1$ $\mathrm{cm}$ down to bone) was obtained for histopathological examination. Microscopically, the lesion showed proliferation of the well-differentiated stratified squamous epithelium, forming endophytic complex branching networks interconnected with multiple deep keratinfilled clefts and crypts. Such characteristic "burrowing" architecture was evident, which resembles rabbit burrows; the hallmark of OCC (Fig. 2A-C). Besides, the neoplastic cells displayed tortuous cyst-like sinuses (Fig. 2D, E), and neoplastic islands of well-differentiated squamous cells showing minimal cellular atypia
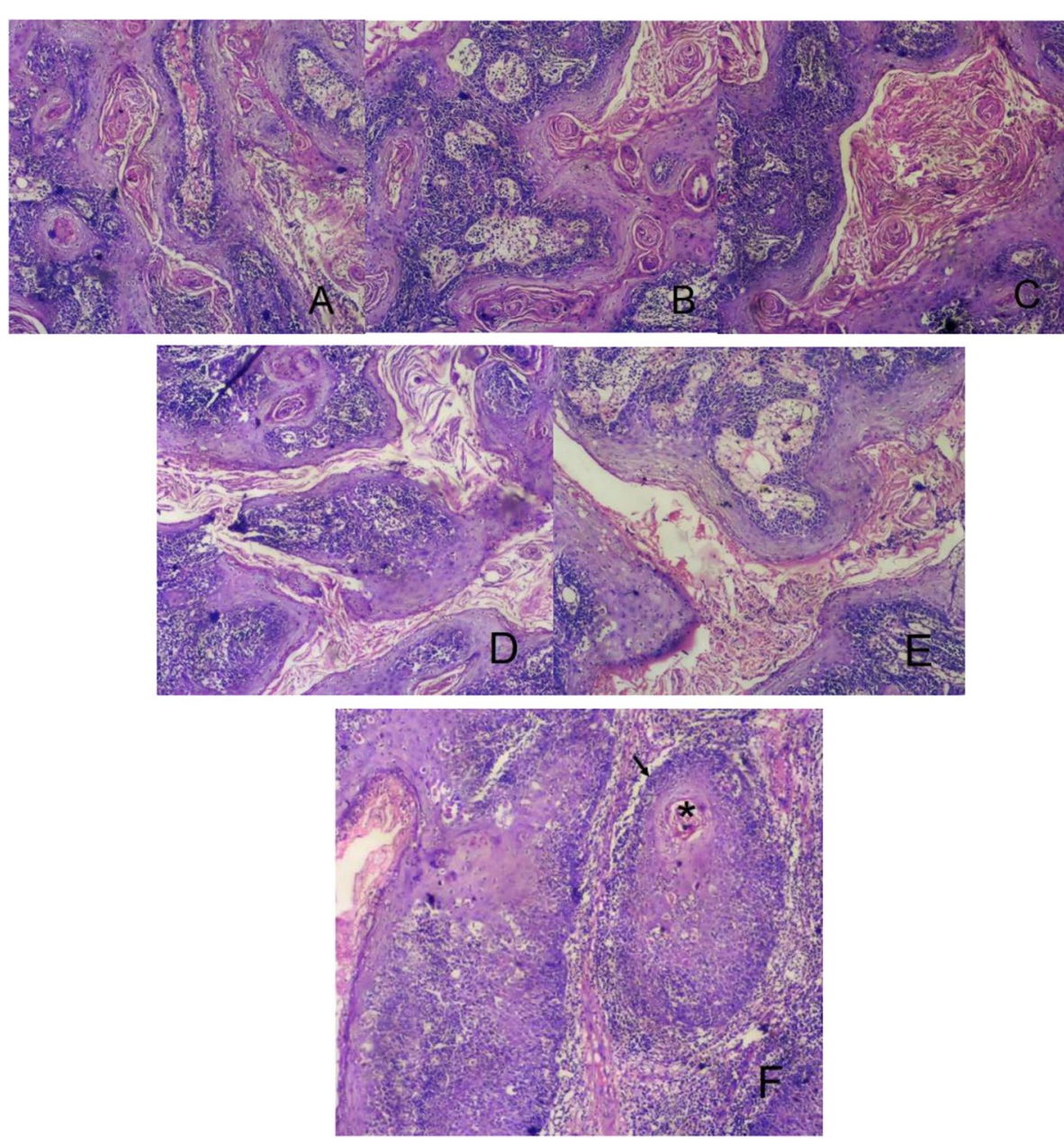

Fig. 2 Photomicrographs, hematoxylin and eosin (H\&E) staining, showing A, B endophytic deep "burrowing" growth of neoplastic epithelium with keratin-filled crypts (x 100). C A higher magnification ( $\times 200)$. D, E Neoplastic cells forming cyst-like sinuses and tracts filled with keratin debris $(\times$ 100). F Neoplastic islands. Arrow points to the tumor nest and ${ }^{*}$ indicates the keratin pearls ( $\left.\times 100\right)$ 
and mitosis, with stromal reaction. Notably, there was a close similarity of this field to the well-differentiated conventional OSCC (Fig. 2F).

Based on the previously mentioned clinicopathological features, a diagnosis of a separate variant of OSCC named OCC was made. The surgical decision was a wide excision as the case showed no bone invasion. Neck dissection was not carried out as there was no clinical indication. Of note, management was hard because of the patient's age.

\section{Discussion}

OCC is a unique variant of OSCC that shows distinct clinicopathological features $[1,8]$. Intraorally, OCC occurs most commonly on mucoperiosteum, with a predilection for mandibular gingiva, as with our patient. Also, it can frequently affect the tongue. This tumor displays variable clinical presentations with/without bone involvement [10]. Unlike conventional OSCC, OCC differs mainly in that it has (1) a locally aggressive behavior with predominantly endophytic "burrowing" growth pattern, including branched sinuses and deep extending keratin-filled crypts and tracts that resemble rabbit burrows. Hence, the term "cuniculatum" derivates from the Latin meaning rabbit burrow crypts (rabbit-hole like) [11, 12], (2) a well-differentiated squamous epithelium with absent or minimal cellular atypia, and (3) a low-grade malignancy that has a better prognosis than conventional OSCC with a lesser tendency to metastasize. Nevertheless, it is crucial to note that the early diagnoses included metastases to local lymph nodes [13].

Unfortunately, its diagnosis is still challenging because OCC is frequently either missed or misdiagnosed and subsequently undertreated. The diagnostic dilemma of this lesion might return to the following factors:

- being unacquainted due to its low incidence in addition to insufficient knowledge about this entity, with consequent under-reporting [14].

- improper biopsy taking (fragmented, limited, or inadequate depth) which may omit the histopathological parameters necessary for reliable diagnosis [10].

To avoid underdiagnosis of OCC and its confusion with other tumors, a diagnostic process is necessary which entails proper knowledge of its entity and diagnostic criteria, an extended clinical evaluation of the suspected cases, following by performance of proper biopsies, appropriate thorough specimen sectioning of these biopsies, and then careful histopathological assessment which is crucial to reveal the endophytic 'burrowing pattern', the hallmark of this lesion. Eventually, proper correlation of clinicopathological findings is essential for confident

Familiarity and awareness of OCC entity

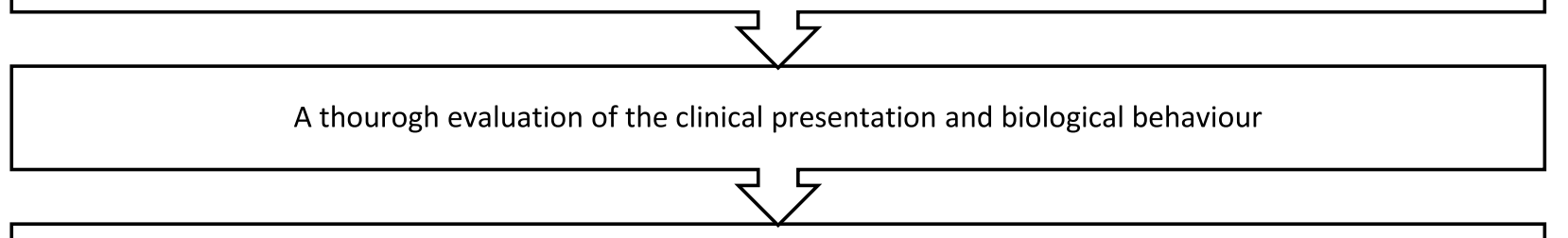

Performance of numerous deep biopsies of the suspected lesion with thorough sampling

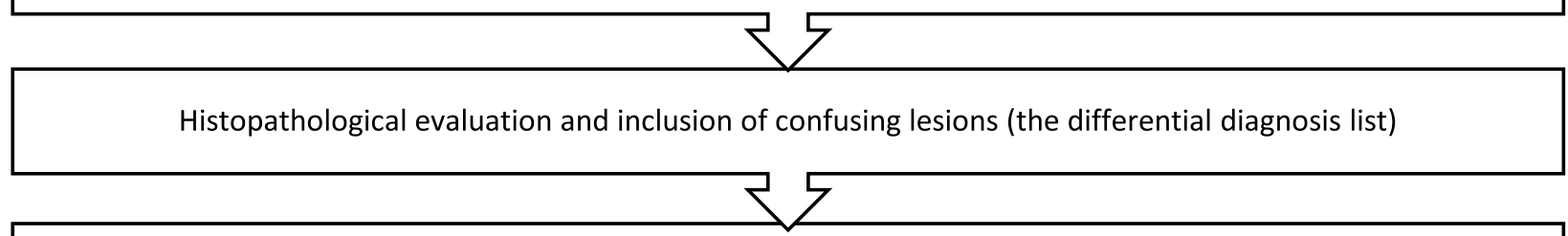

Correlation of clinicopathological findings and the radiographic features (specified by the WHO classification)

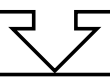

Exclusion of other confusing lesions and diagnosis of OCC

Fig. 3 Basis for a proper approach to diagnose OCC 


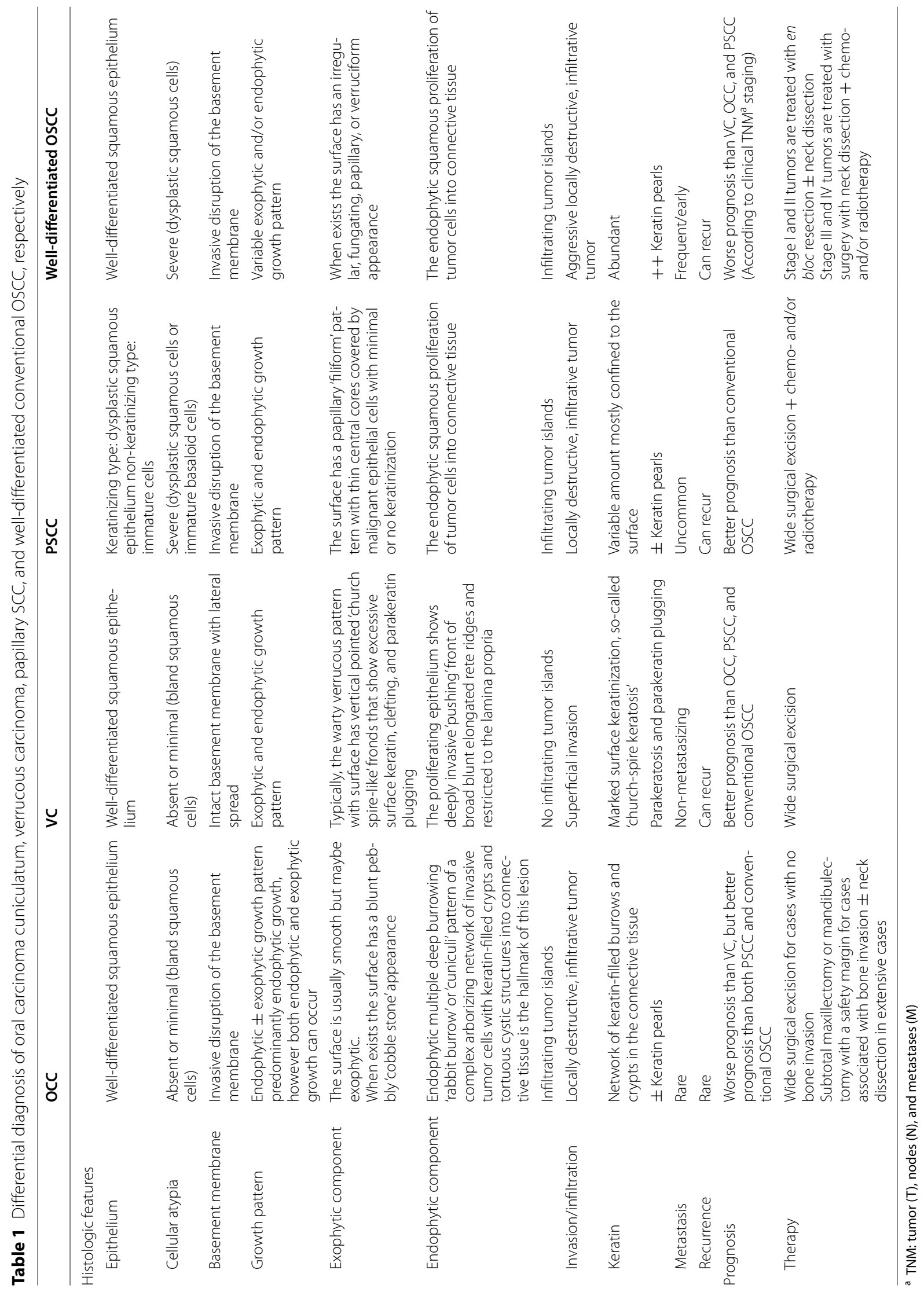


differentiation of OCC from other confusing lesions and in turn for its final diagnosis with the consequent establishment of the proper treatment plan (Fig. 3).

\section{Wide surgical excision for cases with no bone invasion}

The differential diagnosis list of OCC includes lesions of the oral mucosa with exophytic surface and well-differentiated dysplastic epithelial component: VC, papillary squamous cell carcinoma (PSCC), and well-differentiated conventional OSCC. All of these lesions can be distinguished when biopsied, as each variant has a unique histopathological appearance. Consequently, a precise histological diagnosis can assist the clinician in planning accurate treatment, because the prognosis of each varies significantly. The distinctive properties of OCC and other similar lesions to differentiate between them are declared in Table 1.

OCC managed for cases associated with bone involvement is subtotal maxillectomy or mandibulectomy with a safety margin. Meanwhile, wide surgical excision is the treatment of choice for cases with no bone invasion. This tumor is of low risk of metastasis. Neck dissection is only required if lymph node enlargement is clinically evident. The role of radiotherapy and chemotherapy remain questionable in the treatment of OCC [10].

\section{Conclusions}

In conclusion, diagnosis of OCC requires familiarity with the entity, awareness of clinical and additional parameters, passing through obtaining abundant tissues, and ending with proper knowledge of the histopathological evidence that leads to an accurate diagnosis.

\section{Abbreviations}

CC: Carcinoma cuniculatum; VC: Verrucous carcinoma; WHO: World Health Organization; OCC: Oral carcinoma cuniculatum; OSCC: Oral squamous cell carcinoma; H\&E: Hematoxylin and eosin; PSCC: Papillary squamous cell carcinoma; TNM: Tumor, nodes, and metastases.

\section{Acknowledgements}

Not applicable.

\section{Authors' contributions}

All authors analyzed and interpreted the patient data. HA performed the histological examination, and SB was a major contributor in writing the manuscript. AA substantively revised the work. The authors read and approved the manuscript.

\section{Funding}

None.

\section{Availability of data and material}

The data used during the current study are available from the corresponding author on reasonable request.

\section{Declarations}

Ethics approval and consent to participate

Not applicable.

\section{Consent for publication}

Written informed consent was obtained from the patient for publication of this case report and accompanying images before the submission of the manuscript.

\section{Competing interests}

The authors declare that they have no competing interests.

\section{Author details}

${ }^{1}$ Oral Pathology Department, Faculty of Dentistry, British University in Egypt (BUE), El Sherouk City, Suez Desert Road, P.O. Box 43, Cairo 11837, Egypt. ${ }^{2}$ Oral \& Maxillofacial Pathology Department, Faculty of Dentistry, Cairo University, Cairo, Egypt.

Received: 16 September 2021 Accepted: 18 December 2021 Published online: 17 January 2022

\section{References}

1. Barnes L, UniversitätsSpital Zürich, Departement Pathologie, International Academy of Pathology, World Health Organization, International Agency for Research on Cancer, et al. Pathology and genetics of head and neck tumours: tumours of the oral cavity and oropharynx. Lyon: International Agency for Research on Cancer (IARC) Press; 2005. p. 163-75.

2. Aird I, Johnson HD, Lennox B, Stansfeld AG. Epithelioma cuniculatum: a variety of squamous carcinoma peculiar to the foot. Br J Surg. 1954:42(173):245-50.

3. Kao GF, Graham JH, Helwig EB. Carcinoma cuniculatum (verrucous carcinoma of the skin): a clinicopathologic study of 46 cases with ultrastructural observations. Cancer [Internet]. 1982;49(11):2395-403 10.1002/1097-0142(19820601)49:11\%3C2395::AIDCNCR2820491129\%3E3.0.CO;2-3.

4. Lawrence-Brown MMD, Gollow IJ, Lam TP, Frost FA. Carcinoma cuniculatum of the abdominal wall. Med J Aust. 1984;140(11):668-9.

5. Barreto JE, Velazquez EF, Ayala E, Torres J, Cubilla AL. Carcinoma cuniculatum: a distinctive variant of penile squamous cell carcinoma - report of 7 cases. Am J Surg Pathol. 2007 Jan;31(1):71-5.

6. Smyth M, Jaaback K, Tabrizi SN, Garland SM, Yin H, Scurry J. Carcinoma cuniculatum of the cervix. Pathology. 2014;46(4):353-5.

7. Flieger $\mathrm{S}$, Owiński T. Epithelioma cuniculatum an unusual form of mouth and jaw neoplasm. Czas Stomatol. 1977;30(5):395-401.

8. El-Naggar AK, Chan JKC, Takata T, Grandis JR, Slootweg PJ. The fourth edition of the head and neck World Health Organization blue book: editors' perspectives. Vol. 66, Human Pathology. United States; 2017. p. 10-12.

9. Yadav S, Bal M, Rane S, Mittal N, Janu A, Patil A. Carcinoma cuniculatum of the oral cavity: a series of 6 cases and review of literature. Head Neck Pathol. 2021. https://doi.org/10.1007/s12105-021-01340-6.

10. Farag AF, Abou-Alnour DA, Abu-Taleb NS. Oral carcinoma cuniculatum, an unacquainted variant of oral squamous cell carcinoma: a systematic review. Imaging Sci Dent. 2018;48(4):233-44.

11. Thavaraj S, Cobb A, Kalavrezos N, Beale T, Walker DM, Jay A. Carcinoma cuniculatum arising in the tongue. Head Neck Pathol. 2012;6(1):130-4.

12. Padilla RJ, Murrah VA. Carcinoma cuniculatum of the oral mucosa: a potentially underdiagnosed entity in the absence of clinical correlation. Oral Surg Oral Med Oral Pathol Oral Radiol. 2014;118(6):684-93.

13. Sun Y, Kuyama K, Burkhardt A, Yamamoto H. Clinicopathological evaluation of carcinoma cuniculatum: a variant of oral squamous cell carcinoma. J oral Pathol Med. 2012;41(4):303-8.

14. Datar UV, Kale AMD. Oral carcinoma cuniculatum: a new entity in the clinicopathological spectrum of oral squamous cell carcinoma. J Clin Diagnostic Res. 2017;11(1):ZD37-9.

\section{Publisher's Note}

Springer Nature remains neutral with regard to jurisdictional claims in published maps and institutional affiliations. 